\title{
A NUMERICAL SOLUTION FOR A CLASS OF TIME FRACTIONAL DIFFUSION EQUATIONS WITH DELAY
}

\author{
VLADIMIR G. PIMENOV ${ }^{a, b}$, AHMED S. HENDY ${ }^{b, c, *}$
}

${ }^{a}$ Institute of Mathematics and Mechanics

Ural Branch of the Russian Academy of Sciences, 16 Kovalevskoy St., Yekaterinburg 620000, Russia

\author{
${ }^{b}$ Department of Computational Mathematics and Computer Science, Institute of Natural Sciences and Mathematics \\ Ural Federal University, 19 Mira St., Yekaterinburg 620002, Russia \\ e-mail: $v \cdot g \cdot$ pimenov@urfu.ru \\ ${ }^{c}$ Department of Mathematics, Faculty of Science \\ Benha University, Benha 13511, Egypt \\ e-mail: ahmed.hendy@fsc.bu.edu.eg
}

\begin{abstract}
This paper describes a numerical scheme for a class of fractional diffusion equations with fixed time delay. The study focuses on the uniqueness, convergence and stability of the resulting numerical solution by means of the discrete energy method. The derivation of a linearized difference scheme with convergence order $O\left(\tau^{2-\alpha}+h^{4}\right)$ in $L_{\infty}$-norm is the main purpose of this study. Numerical experiments are carried out to support the obtained theoretical results.
\end{abstract}

Keywords: fractional diffusion equation with delay, difference scheme, convergence analysis.

\section{Introduction}

Recently, significantly increased attention regarding partial differential equations which contain fractional derivatives (FPDEs) and integrals has been observed. Due to their ability to model some phenomena more efficiently than partial differential equations with integer derivatives, FPDEs are utilized in many areas of science. Nowadays, the interest of scientists in FPDEs in fields of science and engineering involves anomalous diffusion mechanisms, such as fluid flow in porous materials (Benson et al., 2001), underground environmental problems (Hatano and Hatano, 1998), anomalous transport in biology (Höfling and Franosch, 2013), finance (Raberto et al., 2002; Scalas et al., 2000), viscoelasticity (Bagley and Torvik, 1983), etc., and many other scientific areas. Time delay has been considered in numerous mathematical models, e.g., physiological systems (Batzel and Kappel, 2011), population dynamics (Liu, 2015; Tumwiine et al., 2008) and HIV-infection modeling (Culshaw et al., 2003; Yan and Kou, 2012). Relative controllability and relative

\footnotetext{
${ }^{*}$ Corresponding author
}

constrained controllability of linear fractional systems with delays in the state were discussed by Sikora (2016).

Sufficient conditions for the controllability of linear and nonlinear fractional dynamical systems in finite dimensional spaces were obtained by Balachandran and Kokila (2012). The authors used Schauder fixed point theorem and the controllability Grammian matrix defined by the Mittag-Leffler matrix function. Some theoretical analysis of fractional differential equations with time delay was introduced by Lakshmikantham (2008). Alternative results concerning the existence and attractivity dependence of solutions for a class of non-linear fractional functional differential equations were presented by Chen and Zhou (2011). Some numerical solutions for time delay differential equations were proposed in the literature by means of finite difference methods and others (Bellen and Zennaro, 2003; Jackiewicz et al., 2014; Rihan, 2009; Solodushkin et al., 2017).

Ferreira (2008) studied energy estimates for delay diffusion-reaction. A backward Euler scheme with $L_{2}$-convergence order $O\left(\tau+h^{2}\right)$ was proposed. Zhang 
and Sun (2013) introduced a linearized compact difference scheme for a class of nonlinear delay partial differential equations with initial and Dirichlet boundary conditions. Karatay et al. (2013) predicted an approximation for the time Caputo fractional derivative at time $t_{k+1 / 2}$ with fractional order $0<\alpha<1$. They extended the idea of the Cranck-Nicholson method to time fractional heat equations with convergence order $O\left(\tau^{2-\alpha}+h^{2}\right)$. Some numerical contributions in fractional functional differential equations with delay based on BDF-type shifted Chebyshev polynomials were discussed by Pimenov and Hendy (2015). A numerical solution of a heat conduction equation with delay for the case of a variable coefficient of heat conductivity was proposed by Lekomtsev and Pimenov (2015).

The time fractional reaction diffusion wave equation

$$
\frac{\partial^{\alpha} u(x, t)}{\partial t^{\alpha}}=K \frac{\partial^{2} u(x, t)}{\partial x^{2}}+f(x, t, u(x, t))
$$

has appeared in a broad variety of engineering, biological and physics processes where anomalous diffusion occurs (Wyss, 1986; Schneider and Wyss, 1989), such as those in sub-diffusive or super-diffusive processes.

When $0<\alpha<1$, Eqn. (1) is a time-fractional diffusion equation, while if $1<\alpha<2$, it is a time fractional wave-diffusion equation. In the case where $\alpha=1$, we obtain the classical diffusion equation, and when $\alpha=2$, we obtain the classical wave equation.

Some numerical methods are introduced in the literature for different forms of (1) (Meerschaert and Tadjeran, 2004; Ren and Sun, 2015). Recently, we proposed a difference method for class of non-linear delay distributed order fractional diffusion equations (Pimenov et al., 2017). In this approach, a theoretical analysis of the proposed linear difference scheme is made. Also, a finite difference scheme for semi-linear space-fractional diffusion equations with time delay is given by Hao et al. (2016).

Based on the ideas of Zhang and Sun (2013) and Karatay et al. (2013), we are interested in constructing a linearized difference scheme for (1) which is induced with fixed time delay in the source function as in the simulation of dynamical systems. Specifically, we consider

$$
\frac{\partial^{\alpha} u(x, t)}{\partial t^{\alpha}}=K \frac{\partial^{2} u(x, t)}{\partial x^{2}}+f(x, t, u(x, t), u(x, t-s)),
$$

with the following initial and boundary conditions:

$$
\begin{aligned}
& u(x, t)=\psi(x, t), \quad 0 \leq x \leq L, \quad t \in[-s, 0), \\
& u(0, t)=\phi_{0}(t), \quad u(L, t)=\phi_{L}(t), \quad t>0,
\end{aligned}
$$

where $s>0$ is the delay parameter and $K$ is a positive constant. The fractional derivative is introduced in the
Caputo sense (Miller and Ross, 1993), that is,

$$
\begin{aligned}
&{ }_{0}^{C} D_{t}^{\alpha} u(x, t) \\
& \equiv \frac{\partial^{\alpha} u(x, t)}{\partial t^{\alpha}} \\
&:=\frac{1}{\Gamma(1-\alpha)} \int_{0}^{t}(t-\zeta)^{-\alpha} \frac{\partial u(x, \zeta)}{\partial \zeta} \mathrm{d} \zeta, \\
& 0<\alpha<1 .
\end{aligned}
$$

In this paper, we propose a high-order linearized difference scheme for the time fractional diffusion equation with delay. The degree of complexity is how to approximate the time fractional derivative and the non-linear delay source function. Throughout this work, like Zhang and Sun (2013), we suppose that the function $f(x, t, \mu, \nu)$ and the solution $u(x, t)$ of (2) are sufficiently smooth in the following sense:

- Let $m$ be an integer satisfying $m s \leq T<(m+1) s$. Define $I_{r}=(r s,(r+1) s), \quad r=-1,0, \ldots, m-$ $1, I_{m}=(m s, T), I=\bigcup_{q=-1}^{m} I_{q}$, and assume that $u(x, t) \in C^{(6,2)}([0, L] \times(0, T])$.

- The partial derivatives $f_{\mu}(x, t, \mu, \nu)$ and $f_{\nu}(x, t, \mu, \nu)$ are continuous in the $\epsilon_{0}$-neighborhood of the solution. Define

$$
\begin{gathered}
\left.c_{1}=\sup _{\substack{0<x<L, 0<t \leq T \\
\left|\epsilon_{1}\right| \leq \epsilon_{0},\left|\epsilon_{2}\right| \leq \epsilon_{0}}} \mid f_{\mu}+\epsilon_{1}, u(x, t-s)+\epsilon_{2}\right) \mid, \\
\left.c_{2}=\max _{\substack{0<x<L, 0<t \leq T \\
\left|\epsilon_{1}\right| \leq \epsilon_{0},\left|\epsilon_{2}\right| \leq \epsilon_{0}}} \mid f_{\nu}+\epsilon_{1}, u(x, t-s)+\epsilon_{2}\right) \mid .
\end{gathered}
$$

The rest of this paper is arranged in the following way. We present the derivation of the difference scheme in the following section. Next, in Section 3, the solvability, convergence and stability for the difference scheme are discussed. In Section 4, numerical examples are given to illustrate the accuracy of the presented scheme and to support our theoretical results. Finally, the paper ends with conclusion and some remarks.

\section{Derivation of the difference scheme}

We aim to obtaining a numerical solution based on the Crank-Nicholson method. We need some notation. Take two positive integers $M$ and $n$, let $h=L / M, \tau=s / n$ and write $x_{i}=i h, t_{k}=k \tau$ and $t_{k+1 / 2}=\left(k+\frac{1}{2}\right) \tau=$ $\frac{1}{2}\left(t_{k}+t_{k+1}\right)$. Cover the space-time domain by $\Omega_{h \tau}=$ $\Omega_{h} \times \Omega_{\tau}$, where $\Omega_{h}=\left\{x_{i} \mid 0 \leq i \leq M\right\}, \Omega_{\tau}=\left\{t_{k} \mid-n \leq\right.$ $k \leq N\}, N=\lfloor T / \tau\rfloor$. Let $\mathcal{W}=\left\{\nu \mid \nu=v_{i}^{k}, 0 \leq i \leq\right.$ $M,-n \leq k \leq N\}$ be a grid function space on $\Omega_{h \tau}$. For $\nu \in \mathcal{W}$, we write $v_{i}^{k+1 / 2}=\frac{1}{2}\left(v_{i}^{k}+v_{i}^{k+1}\right)$ and $\delta_{x}^{2} v_{i}^{k}=$ $\left(v_{i+1}^{k}-2 v_{i}^{k}+v_{i-1}^{k}\right) / h^{2}$. 
Lemma 1. (Zhang and Sun, 2013) Let $q(x) \in$ $C^{6}\left[x_{i-1}, x_{i+1}\right]$. Then

$$
\begin{aligned}
& \frac{1}{12}\left(q^{\prime \prime}\left(x_{i-1}\right)+10 q^{\prime \prime}\left(x_{i}\right)+q^{\prime \prime}\left(x_{i+1}\right)\right) \\
& -\frac{1}{h^{2}}\left(q\left(x_{i-1}\right)-2 q\left(x_{i}\right)+q\left(x_{i+1}\right)\right) \\
& =\frac{h^{4}}{240} q^{(6)}\left(\omega_{i}\right),
\end{aligned}
$$

where $\omega_{i} \in\left(x_{i-1}, x_{i+1}\right)$.

We define the grid function on $\Omega_{h \tau}: U(i, k)=$ $u\left(x_{i}, t_{k}\right)$. In the work of Karatay et al. (2013), an approximation to the time Caputo fractional derivative at $t_{k+1 / 2}$ with $0<\alpha_{l}<1$ was given as

$$
\begin{aligned}
& \frac{\partial^{\alpha} u\left(x_{i}, t_{k+1 / 2}\right)}{\partial t^{\alpha}} \\
& =\omega_{1} U_{i}^{k}+\sum_{m=1}^{k-1}\left(\omega_{k-m+1}-\omega_{k-m}\right) u_{i}^{m} \\
& \quad-\omega_{k} U_{i}^{0}+\frac{\sigma}{2^{1-\alpha}}\left(U_{i}^{k+1}-U_{i}^{k}\right)+O\left(\tau^{2-\alpha}\right),
\end{aligned}
$$

where

$$
\begin{gathered}
\omega_{i}=\sigma\left(\left(i+\frac{1}{2}\right)^{1-\alpha}-\left(i-\frac{1}{2}\right)^{1-\alpha}\right), \\
\sigma=\frac{1}{\tau^{\alpha} \Gamma(2-\alpha)}, \quad 0 \leq i \leq M, \quad 0 \leq k \leq N-1 .
\end{gathered}
$$

We are now in a position to apply (4) to (2a) at the points $\left(x_{i}, t_{k+1 / 2}\right)$, and arrive at

$$
\begin{aligned}
& {\left[\omega_{1} U_{i}^{k}+\sum_{m=1}^{k-1}\left(\omega_{k-m+1}-\omega_{k-m}\right) U_{i}^{m}-\omega_{k} U_{i}^{0}\right.} \\
& \left.+\frac{\sigma}{2^{1-\alpha}}\left(U_{i}^{k+1}-U_{i}^{k}\right)+O\left(\tau^{2-\alpha}\right)\right]+O(\Delta \alpha)^{4} \\
& =K \frac{\partial^{2} u\left(x_{i}, t_{k+1 / 2}\right)}{\partial x^{2}} \\
& \quad+f\left(x_{i}, t_{k+1 / 2}, u\left(x_{i}, t_{k+1 / 2}\right)\right. \\
& \left.\quad u\left(x_{i}, t_{k+1 / 2}-s\right)\right) .
\end{aligned}
$$

Lemma 2. For $g=\left(g_{0}, g_{1}, \ldots, g_{M}\right)$, let the linear operator $\mathfrak{A}$ be defined as

$$
\mathfrak{A} g_{i}=\frac{1}{12}\left(g_{i-1}+10 g_{i}+g_{i+1}\right), \quad 1 \leq i \leq M-1 .
$$

Then we have

$$
\begin{aligned}
\mathfrak{A}\left[\omega_{1} U_{i}^{k}+\sum_{m=1}^{k-1}\left(\omega_{k-m+1}-\omega_{k-m}\right) U_{i}^{m}-\omega_{k} U_{i}^{0}\right. \\
\left.+\frac{\sigma}{2^{1-\alpha}}\left(U_{i}^{k+1}-U_{i}^{k}\right)\right] \\
=K \delta_{x}^{2} U_{i}^{k+1 / 2} \\
\quad+\mathfrak{A} f\left(x_{i}, t_{k+1 / 2}, \frac{3}{2} U_{i}^{k}-\frac{1}{2} U_{i}^{k-1}\right. \\
\left.\quad \frac{1}{2} U_{i}^{k+1-n}+\frac{1}{2} U_{i}^{k-n}\right)+R_{i}^{k}
\end{aligned}
$$

where

$$
\begin{gathered}
\left|R_{i}^{k}\right|=O\left(h^{4}+\tau^{2-\alpha}\right), \\
1 \leq i \leq M-1, \quad 0 \leq k \leq N-1 .
\end{gathered}
$$

Proof. We use Taylor expansions

$$
\begin{aligned}
& \frac{\partial^{2} u\left(x_{i}, t_{k+1 / 2}\right)}{\partial x^{2}} \\
& =\left(\frac{\partial^{2} u\left(x_{i}, t_{k}\right)}{\partial x^{2}}+\frac{\partial^{2} u\left(x_{i}, t_{k+1}\right)}{\partial x^{2}}\right)+O\left(\tau^{2}\right), \\
& u\left(x_{i}, t_{k+1 / 2}\right)=U_{i}^{k+1 / 2} \\
& =\frac{3}{2} U_{i}^{k}-\frac{1}{2} U_{i}^{k-1}+O\left(\tau^{2}\right),
\end{aligned}
$$

$$
\begin{aligned}
u\left(x_{i}, t_{k+1 / 2}-s\right) & =U_{i}^{k-n+\frac{1}{2}} \\
& =\frac{1}{2} U_{i}^{k+1-n}+\frac{1}{2} U_{i}^{k-n}+O\left(\tau^{2}\right),
\end{aligned}
$$

in (7) and obtain

$$
\begin{aligned}
& {\left[\omega_{1} U_{i}^{k}+\sum_{m=1}^{k-1}\left(\omega_{k-m+1}-\omega_{k-m}\right) U_{i}^{m}-\omega_{k} U_{i}^{0}\right.} \\
& \left.+\frac{\sigma}{2^{1-\alpha}}\left(U_{i}^{k+1}-U_{i}^{k}\right)+O\left(\tau^{2-\alpha}\right)\right] \\
& =\frac{K}{2}\left(\frac{\partial^{2} u\left(x_{i}, t_{k}\right)}{\partial x^{2}}+\frac{\partial^{2} u\left(x_{i}, t_{k+1}\right)}{\partial x^{2}}\right)+O\left(\tau^{2}\right) \\
& \quad+f\left(x_{i}, t_{k+1 / 2}, \frac{3}{2} U_{i}^{k}-\frac{1}{2} U_{i}^{k-1},\right. \\
& \left.\quad \frac{1}{2} U_{i}^{k+1-n}+\frac{1}{2} U_{i}^{k-n}\right),
\end{aligned}
$$

where we use the continuity of the derivatives of $f$ in its 
third and fourth components. We rewrite this as

$$
\begin{aligned}
& {\left[\omega_{1} U_{i}^{k}+\sum_{m=1}^{k-1}\left(\omega_{k-m+1}-\omega_{k-m}\right) U_{i}^{m}-\omega_{k} U_{i}^{0}\right.} \\
& \left.+\frac{\sigma}{2^{1-\alpha}}\left(U_{i}^{k+1}-U_{i}^{k}\right)\right]+O\left(\tau^{2-\alpha}\right) \\
& =\frac{K}{2}\left(\frac{\partial^{2} u\left(x_{i}, t_{k}\right)}{\partial x^{2}}+\frac{\partial^{2} u\left(x_{i}, t_{k+1}\right)}{\partial x^{2}}\right) \\
& \quad+f\left(x_{i}, t_{k+1 / 2}, \frac{3}{2} U_{i}^{k}-\frac{1}{2} U_{i}^{k-1},\right. \\
& \left.\quad \frac{1}{2} U_{i}^{k+1-n}+\frac{1}{2} U_{i}^{k-n}\right)+O\left(\tau^{2}\right),
\end{aligned}
$$

According to Lemma 1 we have

$$
\begin{aligned}
\mathfrak{A} \frac{\partial^{2} u\left(x_{i}, t_{k}\right)}{\partial x^{2}}=\delta_{x}^{2} U_{i}^{k}+\frac{h^{4}}{240} \frac{\partial^{6} u}{\partial x^{6}}\left(\theta_{i}^{k}, t_{k}\right), & \\
\theta_{i}^{k} & \in\left(x_{i-1}, x_{i+1}\right) .
\end{aligned}
$$

Thus, applying $\mathfrak{A}$ to 10 , we arrive at

$$
\begin{aligned}
\mathfrak{A}\left[\omega_{1} U_{i}^{k}+\sum_{m=1}^{k-1}\left(\omega_{k-m+1}-\omega_{k-m}\right) u_{i}^{m}-\omega_{k} U_{i}^{0}\right. \\
\left.+\frac{\sigma}{2^{1-\alpha}}\left(U_{i}^{k+1}-U_{i}^{k}\right)\right] \\
=K \delta_{x}^{2} U_{i}^{k+1 / 2} \\
\quad+\mathfrak{A} f\left(x_{i}, t_{k+1 / 2}, \frac{3}{2} U_{i}^{k}-\frac{1}{2} U_{i}^{k-1},\right. \\
\left.\quad \frac{1}{2} U_{i}^{k+1-n}+\frac{1}{2} U_{i}^{k-n}\right)+O\left(\tau^{2-\alpha}+h^{4}\right)
\end{aligned}
$$

as $u(x, t) \in C^{(6,2)}(I \times(0, T])$.

The final form of our difference scheme is obtained by neglecting $R_{i}^{k}$ and replacing $U_{i}^{k}$ with $u_{i}^{k}$ in (8):

$$
\begin{aligned}
& \mathfrak{A}\left[\omega_{1} u_{i}^{k}+\sum_{m=1}^{k-1}\left(\omega_{k-m+1}-\omega_{k-m}\right) u_{i}^{m}-\omega_{k} u_{i}^{0}\right. \\
& \left.+\frac{\sigma^{l}}{2^{1-\alpha_{l}}}\left(u_{i}^{k+1}-u_{i}^{k}\right)\right] \\
& =K \delta_{x}^{2} u_{i}^{k+1 / 2}+\mathfrak{A} f\left(x_{i}, t_{k+1 / 2}, \frac{3}{2} u_{i}^{k}-\frac{1}{2} u_{i}^{k-1},\right. \\
& \left.\quad \frac{1}{2} u_{i}^{k+1-n}+\frac{1}{2} u_{i}^{k-n}\right),
\end{aligned}
$$

and supplying appropriate initial and boundary conditions:

$$
\begin{aligned}
& u_{0}^{k}=\phi_{0}\left(t_{k}\right), \quad u_{M}^{k}=\phi_{L}\left(t_{k}\right), \quad 1 \leq k \leq N, \\
& u_{i}^{k}=\psi\left(x_{i}, t_{k}\right), \quad 0 \leq i \leq M, \quad-n \leq k \leq 0 .
\end{aligned}
$$

\section{Analysis of the difference scheme}

Before introducing the uniqueness, convergence and stability theorems in $L_{\infty}$ norm for the proposed difference scheme using a discrete energy method, we introduce some notation.

If the spatial domain $[0, L]$ is covered by $\Omega_{h}=$ $\left\{x_{i} \mid 0 \leq i \leq M,\right\}$, let $V_{h}=\{v \mid v=$ $\left.\left(v_{0}, \ldots, v_{M}\right), \quad v_{0}=v_{M}=0\right\}$ be a grid function space on $\Omega_{h}$. For any $u, v \in V_{h}$, introduce the discrete inner products and corresponding norms as

$$
\begin{gathered}
\langle u, v\rangle=-h \sum_{i=1}^{M-1}\left(\mathfrak{A} u_{i}\right)\left(\delta_{x}^{2} v_{i}\right) \\
=h \sum_{i=1}^{M-1}\left(\delta_{x} u_{i+1 / 2}\right)\left(\delta_{x} v_{i+1 / 2}\right) \\
-\frac{h^{2}}{12} h \sum_{i=1}^{M-1}\left(\delta_{x}^{2} u_{i}\right)\left(\delta_{x}^{2} v_{i}\right), \\
|u|_{1}^{2}=h \sum_{i=1}^{M}\left(\delta_{x} u_{i-1 / 2}\right)^{2}, \\
\|u\|^{2}=h \sum_{i=1}^{M-1}\left(u_{i}\right)^{2}, \quad\|u\|_{\infty}=\max _{1 \leq i \leq M-1}\left|u_{i}\right| .
\end{gathered}
$$

According to Samarskii and Andreev (1976) or Zhang and Sun (2013), for any $u \in V_{h}$ the following inequalities are fulfilled:

$$
\begin{gathered}
\frac{2}{3}|u|_{1}^{2} \leq\langle u, u\rangle \leq|u|_{1}^{2}, \\
\|u\|_{\infty} \leq \frac{\sqrt{L}}{2}|u|_{1}, \quad\|u\|^{2} \leq \frac{L}{6}|u|_{1}^{2} .
\end{gathered}
$$

It is directly observed from (12) that

$$
\|u\|^{2} \leq \frac{L^{2}}{4}\langle u, u\rangle, \quad\|u\|_{\infty}^{2} \leq \frac{3 L}{8}\langle u, u\rangle .
$$

Lemma 3. For any $v \in V_{h}$, we have $\|\mathfrak{A} v\|^{2} \leq\|v\|^{2}$.

Lemma 4. For any $u, v \in V_{h}$, we have

$$
-h \sum_{i=1}^{M-1}\left(\delta_{x}^{2} u_{i}\right) v_{i}=h \sum_{i=1}^{M}\left(\delta_{x} u_{i-1 / 2}\right)\left(\delta_{x} v_{i-1 / 2}\right) .
$$

For the ease of further analysis, Eqn. (4) can be rewritten as

$$
\begin{aligned}
& \frac{\partial^{\alpha} u\left(x_{i}, t_{k+1 / 2}\right)}{\partial t^{\alpha}} \\
& =\frac{\tau^{1-\alpha}}{\Gamma(2-\alpha)}\left[a_{k-m+1}^{\alpha} u_{t, m-1}+a_{0}^{\alpha} u_{t, k}\right] \\
& \quad+O\left(\tau^{2-\alpha}\right)
\end{aligned}
$$

such that

$$
a_{0}^{\alpha}=\left(\frac{1}{2}\right)^{1-\alpha},
$$




$$
a_{l}^{\alpha}=(l+1 / 2)^{1-\alpha}-(l-1 / 2)^{1-\alpha}, \quad l \geq 1 .
$$

Then

$$
\frac{\partial^{\alpha} u\left(x_{i}, t_{k+1 / 2}\right)}{\partial t^{\alpha}}=\frac{\tau^{1-\alpha}}{\Gamma(2-\alpha)} \sum_{m=0}^{k} C_{k-m}^{(k+1)} u_{t, m},
$$

such that

$$
u_{t, m}=\frac{u_{m+1}-u_{m}}{\tau},
$$

where $c_{0}^{(k+1)}=a_{0}^{\alpha}$ for $j=0$ and for $j \geq 1$ we have

$$
C_{m}^{(k+1)}= \begin{cases}a_{0}^{\alpha}, & m=0 \\ a_{m}^{\alpha}, & 1 \leq m \leq k-1, \\ a_{k}^{\alpha}, & m=k\end{cases}
$$

Then at $0<\alpha \leq 1$ and for $u(x, t) \in C^{2}[0, T]$, we have

$$
\begin{aligned}
& \frac{\partial^{\alpha} u\left(x_{i}, t_{k+1 / 2}\right)}{\partial t^{\alpha}} \\
& =\sum_{n=0}^{k} g_{n}^{(k+1)}\left[u\left(x_{i}, t_{n+1}\right)-u\left(x_{i}, t_{n}\right)\right]+O\left(\tau^{2-\alpha}\right) \\
& :=\Delta_{t_{k+1 / 2}}^{\alpha} u+O\left(\tau^{2-\alpha}\right)
\end{aligned}
$$

such that

$$
g_{n}^{(k+1)}=\frac{\tau^{-\alpha}}{\Gamma(2-\alpha)} C_{k-n}^{(k+1)}
$$

Lemma 5. For any $0<\alpha<1, C_{m}^{(k+1)}(0 \leq m \leq k, k \geq$ $1)$, and if $3^{\alpha} \geq 3 / 2$, we have

$$
\begin{gathered}
C_{k}^{k+1}>\frac{1-\alpha}{2}(k+1 / 2)^{-\alpha}, \\
C_{0}^{(k+1)}>C_{1}^{(k+1)}>\cdots>C_{k-1}^{(k+1)}>C_{k}^{k+1} .
\end{gathered}
$$

Proof. For $k \geq 1$, we get

$$
\begin{aligned}
C_{k}^{(k+1)} & =(1 / 2)^{1-\alpha}\left[(2 k+1)^{1-\alpha}-(2 k-1)^{1-\alpha}\right] \\
& >\frac{1-\alpha}{2}\left(\frac{1}{2}\right)^{-\alpha} \int_{0}^{1} \frac{\mathrm{d} \eta}{(2 k+1-\eta)^{\alpha}} \\
& >\frac{1-\alpha}{2}\left(\frac{1}{2}\right)^{-\alpha}(2 k+1)^{-\alpha} \\
& >\frac{1-\alpha}{2}\left(k+\frac{1}{2}\right)^{-\alpha} .
\end{aligned}
$$

Moreover

$$
C_{0}^{(k+1)}=(1 / 2)^{1-\alpha}>\frac{1-\alpha}{2}\left(\frac{1}{2}\right)^{-\alpha},
$$

so that 17 is achieved. Observe that $C_{1}^{(k+1)}>\cdots>$ $C_{k-1}^{(k+1)}>C_{k}^{k+1}$, because we have $a_{l}^{\alpha}>a_{l+1}^{\alpha}, \quad l \geq 1$. Accordingly, the inequality (18) is achieved if $a_{0}^{\alpha} \geq a_{1}^{\alpha}$, which is equivalent to $3^{\alpha} \geq 3 / 2$.
Lemma 6. From Lemma 5 it follows that

$$
g_{k}^{(k+1)}>g_{k-1}^{(k+1)}>\cdots>g_{1}^{(k+1)}>g_{0}^{(k+1)}
$$

and

$$
\begin{aligned}
g_{0}^{(k+1)} & =\frac{\tau^{-\alpha} C_{k}^{(k+1)}}{\Gamma(2-\alpha)} \\
& \geq \frac{\frac{1-\alpha}{2}(k+1 / 2)^{-\alpha}}{\tau^{\alpha} \Gamma(2-\alpha)} \\
& \geq \frac{1-\alpha}{2 T^{\alpha} \Gamma(2-\alpha)}=k_{0} .
\end{aligned}
$$

Lemma 7. (Alikhanov, 2015) If

$$
\begin{aligned}
\left\{g_{k}^{(k+1)}>g_{k-1}^{(k+1)}>\cdots>g_{0}^{(k+1)}>0\right. & \\
& k=0,1, \ldots, M-1\}
\end{aligned}
$$

then for any function $\nu(t)$ defined on the mesh $\left\{t_{k}: t_{0}<\right.$ $\left.t_{1}<t_{2}<\ldots t_{M-1}<t_{M}=T\right\}$, we have

$$
\begin{aligned}
& \nu^{k+1} \Delta_{t_{k+1 / 2}}^{\alpha} \nu \\
& \quad \geq \frac{1}{2} \Delta_{t_{k+1 / 2}}^{\alpha}(\nu)^{2}+\frac{1}{2 g_{k}^{(k+1)}}\left(\Delta_{t_{k+1 / 2}}^{\alpha} \nu\right)^{2}
\end{aligned}
$$

$$
\begin{aligned}
& \nu^{k} \Delta_{t_{k+1 / 2}}^{\alpha} \nu \\
& \geq \frac{1}{2} \Delta_{t_{k+1 / 2}}^{\alpha}(\nu)^{2}-\frac{1}{2\left(g_{k}^{(k+1)}-g_{k-1}^{(k+1)}\right)}\left(\Delta_{t_{k+1 / 2}}^{\alpha} \nu\right)^{2} .
\end{aligned}
$$

Based on Lemma 7, we can deduce the following direct result.

\section{Lemma 8. If}

$$
\begin{aligned}
\left\{g_{k}^{(k+1)}>g_{k-1}^{(k+1)}>\cdots>g_{0}^{(k+1)}>0\right. & \\
& k=0,1, \ldots, M-1\}
\end{aligned}
$$

then for any function $\nu(t)$ defined on the mesh $\left\{t_{k}: t_{0}<\right.$ $\left.t_{1}<t_{2}<\ldots t_{M-1}<t_{M}=T\right\}$ we have the following inequality:

$$
\left(\frac{1}{2} \nu^{k+1}+\frac{1}{2} \nu^{k}\right) \Delta_{t_{k+1 / 2}}^{\alpha} \nu \geq \frac{1}{2} \Delta_{t_{k+1 / 2}}^{\alpha}(\nu)^{2} .
$$

Lemma 9. (Special Gronwall inequality) (Holte, 2009; Kruse and Scheutzow, 2016) Let $z_{k}$ and $g_{k}$ be nonnegative sequences and such that $K$ is a non-negative constant. If

$$
z_{k} \leq K \sum_{0 \leq i<k} g_{i} z_{i}, \quad k \geq 0
$$

then

$$
z_{k} \leq K \exp \left(\sum_{0 \leq j<k} g_{j}\right), \quad k \geq 0 .
$$


We start to prove that our difference scheme admits a unique solution. Next we show that the obtained solution solves (2).

Theorem 1. The difference scheme (11) is uniquely solvable.

Proof. Suppose that $u_{i}^{k}, \quad 0 \leq i \leq M$, is the solution for the obtained difference scheme (11). Using the mathematical induction, the base step is fulfilled from the initial condition (11c) as the solution $u_{i}^{k}$ is determined for $-n \leq k \leq 0$. For the inductive hypothesis, let $u_{i}^{k}$ be determined when $k=l$; then from (11a) we obtain a system of linear algebraic equations with respect to $u_{i}^{l}$. The proof ends by the inductive step as the coefficient matrix of this system is strictly diagonally dominant, so there exists a unique solution $u_{i}^{l+1}$.

We can arrange the system (11) as follows:

$$
\begin{aligned}
& \left(\left[\frac{\sigma}{2^{1-\alpha}}-\frac{K}{2 h^{2}}\right] u_{i+1}^{k+1}+\left[\frac{10}{12} \frac{\sigma}{2^{1-\alpha}}+\frac{K}{h^{2}}\right] u_{i}^{k+1}\right. \\
& \left.+\left[\frac{1}{12} \frac{\sigma}{2^{1-\alpha}}-\frac{K}{2 h^{2}}\right] u_{i-1}^{k+1}\right) \\
& +\left(\left[\frac{1}{12}\left(\omega_{1}-\frac{\sigma}{2^{1-\alpha}}\right)-\frac{K}{2 h^{2}}\right] u_{i+1}^{k}\right. \\
& +\left[\frac{10}{12}\left(\omega_{1}-\frac{\sigma}{2^{1-\alpha}}\right)+\frac{K}{h^{2}}\right] u_{i}^{k} \\
& \left.+\left[\frac{1}{12}\left(\omega_{1}-\frac{\sigma}{2^{1-\alpha}}\right)-\frac{K}{2 h^{2}}\right] u_{i-1}^{k}\right) \\
& +\mathfrak{A}\left(\sum_{m=1}^{k-1}\left(\omega_{k-m+1}-\omega_{k-m}\right) u_{i}^{m}-\omega_{k} u_{i}^{0}\right) \\
& =\mathfrak{A} f\left(x_{i}, t_{k+1 / 2}, \frac{3}{2} u_{i}^{k}-\frac{1}{2} u_{i}^{k-1},\right. \\
& \left.\quad \frac{1}{2} u_{i}^{k+1-n}+\frac{1}{2} u_{i}^{k-n}\right) .
\end{aligned}
$$

According to the system above, the coefficient matrix $\mathrm{A}=$ $\left(a_{i j}\right)$ is strictly diagonally dominant because

$$
\begin{gathered}
\left|a_{i i}\right| \geq \sum_{j \neq i}\left|a_{i j}\right|, \\
a_{i i}=\frac{10}{12} \frac{\sigma}{2^{1-\alpha}}+\frac{K}{h^{2}}, \\
a_{i+1, i}=\frac{1}{12} \frac{\sigma}{2^{1-\alpha}}-\frac{K}{2 h^{2}} \\
=a_{i-1, i}, \quad \frac{\sigma^{l}}{2^{1-\alpha}}>0 .
\end{gathered}
$$

Therefore, the coefficient matrix is nonsingular and this proves the theorem.
Theorem 2. (Convergence theorem) Let $u(x, t) \in$ $C^{6,2}([0, L] \times(-s, T])$ be the solution of (2) such that $u\left(x_{i}, t_{k}\right)=U_{i}^{k}$ and $u_{i}^{k}(0 \leq i \leq M,-n \leq k \leq N)$ is the solution of the difference scheme (11). Write $e_{i}^{k}=$ $U_{i}^{k}-u_{i}^{k}$ for $0 \leq i \leq M,-n \leq k \leq N$. Then if

$$
\tau \leq \tau_{0}=\left(\frac{\epsilon_{0}}{4 C}\right)^{\frac{1}{2-\alpha}}, \quad h \leq h_{0}=\left(\frac{\epsilon_{0}}{4 C}\right)^{\frac{1}{4}}
$$

we have

$$
\left\|e^{k}\right\|_{\infty} \leq \bar{C}\left(\tau^{2-\alpha}+h^{4}\right), \quad 0 \leq k \leq N
$$

where $\bar{C}$ is a positive constant independent of $h$ and $\tau$.

Proof. The difference scheme in (8) and 11a can be rewritten in terms of 16 as follows:

$$
\begin{aligned}
& \mathfrak{A}[\left.\sum_{n=0}^{k} g_{n}^{(k+1)}\left(U_{i}^{n+1}-U_{i}^{n}\right)\right] \\
&= K \delta_{x}^{2} U_{i}^{k+1 / 2}+\mathfrak{A} f\left(x_{i}, t_{k+1 / 2}, \frac{3}{2} U_{i}^{k}-\frac{1}{2} U_{i}^{k-1},\right. \\
&\left.\quad \frac{1}{2} U_{i}^{k+1-n}+\frac{1}{2} U_{i}^{k-n}\right)+R_{i}^{k+1 / 2}, \\
& \mathfrak{A}\left[\sum_{n=0}^{k} g_{n}^{(k+1)}\left(u_{i}^{n+1}-u_{i}^{n}\right)\right] \\
&=K \delta_{x}^{2} u_{i}^{k+1 / 2}+\mathfrak{A} f\left(x_{i}, t_{k+1 / 2}, \frac{3}{2} u_{i}^{k}-\frac{1}{2} u_{i}^{k-1},\right. \\
&\left.\quad \frac{1}{2} u_{i}^{k+1-n}+\frac{1}{2} u_{i}^{k-n}\right) .
\end{aligned}
$$

The error difference scheme can be obtained by subtracting (22) from (21), the latter with $u$ replaced by $U$, as follows:

$$
\begin{aligned}
\mathfrak{A} & {\left[\sum_{n=0}^{k} g_{n}^{(k+1)}\left(e_{i}^{n+1}-e_{i}^{n}\right)\right] } \\
= & K \delta_{x}^{2} e_{i}^{k+1 / 2}+R_{i}^{k+1 / 2}+\mathfrak{A}\left[f \left(x_{i}, t_{k+1 / 2}, \frac{3}{2} U_{i}^{k}\right.\right. \\
& \left.-\frac{1}{2} U_{i}^{k-1}, \frac{1}{2} U_{i}^{k+1-n}+\frac{1}{2} U_{i}^{k-n}\right) \\
& -f\left(x_{i}, t_{k+1 / 2}, \frac{3}{2} u_{i}^{k}-\frac{1}{2} u_{i}^{k-1}\right. \\
& \left.\left.\frac{1}{2} u_{i}^{k+1-n}+\frac{1}{2} u_{i}^{k-n}\right)\right]
\end{aligned}
$$

and

$$
\begin{gathered}
e_{0}^{k}=0, \quad e_{M}^{k}=0, \quad 1 \leq k \leq N, \\
e_{i}^{k}=0, \quad 0 \leq i \leq M, \quad-n \leq k \leq 0 .
\end{gathered}
$$


Multiplying (23) by $-h\left(\delta_{x}^{2} e_{i}^{k+1 / 2}\right)$ and summing up for $i$ from 1 to $M-1$ yields

$$
\begin{aligned}
-h & \sum_{i=1}^{M-1} \mathfrak{A}\left[\sum_{n=0}^{k} g_{n}^{(k+1)}\left(e_{i}^{n+1}-e_{i}^{n}\right)\right] \delta_{x}^{2} e_{i}^{k+1 / 2} \\
= & -K\left\|\delta_{x}^{2} e_{i}^{k+1 / 2}\right\|^{2}-h \sum_{i=1}^{M-1}\left(R_{i}^{k+1 / 2}\right) \delta_{x}^{2} e^{k+1 / 2} \\
& -h \sum_{i=1}^{M-1} \mathfrak{A}\left[f \left(x_{i}, t_{k+1 / 2}, \frac{3}{2} U_{i}^{k}-\frac{1}{2} U_{i}^{k-1},\right.\right. \\
& \left.\frac{1}{2} U_{i}^{k+1-n}+\frac{1}{2} U_{i}^{k-n}\right) \\
& -f\left(x_{i}, t_{k+1 / 2}, \frac{3}{2} u_{i}^{k}-\frac{1}{2} u_{i}^{k-1}\right. \\
& \left.\left.\frac{1}{2} u_{i}^{k+1-n}+\frac{1}{2} u_{i}^{k-n}\right)\right] \delta_{x}^{2} e_{i}^{k+1 / 2} .
\end{aligned}
$$

We will prove (20) by strong mathematical induction. The base case is evident: following (25), it is clear that $\left\|e^{k}\right\|_{\infty}=0, \quad-n \leq k \leq 0$, so in particular we have $\left\|e^{0}\right\|_{\infty}=0$.

Next, suppose that (20) is fulfilled for $0 \leq k \leq \ell$; then we will show that 20 holds for $k=\ell+1$.

From the inductive hypothesis, and when $\tau$ and $h$ satisfy (19), we obtain

$$
\left\|e^{k}\right\|_{\infty} \leq C\left(\tau^{2-\alpha}+h^{4}\right) \leq \frac{\epsilon_{0}}{2}, \quad 0 \leq k \leq \ell .
$$

From (27), we conclude that $\left|e^{k}\right| \leq \epsilon_{0} / 2, \quad 0 \leq k \leq \ell$, and so $\left|U_{i}^{k}-u_{i}^{k}\right| \leq \epsilon_{0} / 2,\left|U_{i}^{k-1}-u_{i}^{k-1}\right| \leq \epsilon_{0} / 2, \quad 0 \leq$ $k \leq \ell$. Then $\left|\frac{3}{2}\left(U_{i}^{k}-u_{i}^{k}\right)-\frac{1}{2}\left(U_{i}^{k-1}-u_{i}^{k-1}\right)\right| \leq \epsilon_{0} / 2$, and the following inequality is fulfilled $\mid\left(\frac{3}{2} U_{i}^{k}-\frac{1}{2} U_{i}^{k-1}\right)-$ $\left(\frac{3}{2} u_{i}^{k}-\frac{1}{2} u_{i}^{k-1}\right) \mid \leq \epsilon_{0}, \quad 0 \leq i \leq M, \quad 0 \leq k \leq$ $\ell$. In the same way, we conclude that $\mid \frac{1}{2}\left(U_{i}^{k+1-n}-\right.$ $\left.u_{i}^{k+1-n}\right)+\frac{1}{2}\left(U_{i}^{k-n}-u_{i}^{k-n}\right) \mid \leq \epsilon_{0} / 2$. Then the following inequality is obtained: $\mid\left(\frac{1}{2} U_{i}^{k+1-n}+\frac{1}{2} U_{i}^{k-n}\right)-$ $\left(\frac{1}{2} u_{i}^{k+1-n}+\frac{1}{2} u_{i}^{k-n}\right) \mid \leq \epsilon_{0}, \quad 0 \leq i \leq M, \quad 0 \leq k \leq \ell$. Consequently,

$$
\begin{gathered}
\mid f\left(x_{i}, t_{k+1 / 2}, \frac{3}{2} U_{i}^{k}-\frac{1}{2} U_{i}^{k-1}, \frac{1}{2} U_{i}^{k+1-n}+\frac{1}{2} U_{i}^{k-n}\right) \\
-f\left(x_{i}, t_{k+1 / 2}, \frac{3}{2} u_{i}^{k}-\frac{1}{2} u_{i}^{k-1}, \frac{1}{2} u_{i}^{k+1-n}+\frac{1}{2} u_{i}^{k-n}\right) \mid \\
\leq c_{1}\left|\frac{3}{2} e_{i}^{k}-\frac{1}{2} e_{i}^{k-1}\right|+c_{2}\left|\frac{1}{2} e_{i}^{k+1-n}+\frac{1}{2} e_{i}^{k-n}\right|,
\end{gathered}
$$

and then

$$
\begin{aligned}
\mid \mathfrak{A}\left[f\left(x_{i}, t_{k+1 / 2}, \frac{3}{2} U_{i}^{k}-\frac{1}{2} U_{i}^{k-1}, \frac{1}{2} U_{i}^{k+1-n}+\frac{1}{2} U_{i}^{k-n}\right)\right. \\
\left.-f\left(x_{i}, t_{k+1 / 2}, \frac{3}{2} u_{i}^{k}-\frac{1}{2} u_{i}^{k-1}, \frac{1}{2} u_{i}^{k+1-n}+\frac{1}{2} u_{i}^{k-n}\right)\right] \mid \\
\leq \mathfrak{A}\left(c_{1}\left|\frac{3}{2} e_{i}^{k}-\frac{1}{2} e_{i}^{k-1}\right|+c_{2}\left|\frac{1}{2} e_{i}^{k+1-n}+\frac{1}{2} e_{i}^{k-n}\right|\right),
\end{aligned}
$$

where $0 \leq i \leq M, \quad 0 \leq k \leq \ell$.

Now, we will deal with each part of (26) individually,

$$
\begin{aligned}
\eta_{1} & :=-h \sum_{i=1}^{M-1} \mathfrak{A}\left[\sum_{n=0}^{k} g_{n}^{(k+1)}\left(e_{i}^{n+1}-e_{i}^{n}\right)\right] \delta_{x}^{2} e_{i}^{k+1 / 2} \\
& =\sum_{n=0}^{k} g_{n}^{(k+1)}\left\langle e^{n+1}-e^{n}, e^{k+1 / 2}\right\rangle .
\end{aligned}
$$

Using Lemma 8 in 29], we obtain

$$
\eta_{1} \geq \frac{1}{2} \sum_{n=0}^{k} g_{n}^{(k+1)}\left(\left\langle e^{n+1}, e^{n+1}\right\rangle-\left\langle e^{n}, e^{n}\right\rangle\right) .
$$

By the Cauchy-Schwarz inequality, we get

$$
\begin{aligned}
\eta_{2} & :=-h \sum_{i=1}^{M-1}\left(R_{i}^{k+1 / 2}\right) \delta_{x}^{2} e^{k+1 / 2} \\
& \leq \frac{K}{2} \mid \delta_{x}^{2} e^{k+1 / 2}\left\|^{2}+\frac{1}{2 K}\right\| R^{k+1 / 2} \|^{2} .
\end{aligned}
$$

Moreover,

$$
\eta_{3}:=-h \sum_{i=1}^{M-1} \mathfrak{A} \varrho_{i}^{k+1 / 2} \delta_{x}^{2} e_{i}^{k+1 / 2},
$$

such that

$$
\begin{aligned}
\varrho_{i}^{k+1 / 2} & \\
= & {\left[f \left(x_{i}, t_{k+1 / 2}, \frac{3}{2} U_{i}^{k}-\frac{1}{2} U_{i}^{k-1},\right.\right.} \\
& \left.\frac{1}{2} U_{i}^{k+1-n}+\frac{1}{2} U_{i}^{k-n}\right) \\
& \left.-f\left(x_{i}, t_{k+1 / 2}, \frac{3}{2} u_{i}^{k}-\frac{1}{2} u_{i}^{k-1}, \frac{1}{2} u_{i}^{k+1-n}+\frac{1}{2} u_{i}^{k-n}\right)\right]
\end{aligned}
$$

and

$$
\left\|R^{k+1 / 2}\right\|^{2} \leq L c_{3}^{2}\left(\tau^{2-\alpha}+h^{4}\right)^{2} .
$$

Using (28), we can predict that

$$
\begin{aligned}
\eta_{3} \leq & \left\langle\mathfrak { A } \left( c_{1}\left|\frac{3}{2} e_{i}^{k}-\frac{1}{2} e_{i}^{k-1}\right|+c_{2} \mid \frac{1}{2} e_{i}^{k+1-n}\right.\right. \\
& \left.\left.+\frac{1}{2} e_{i}^{k-n} \mid\right), \delta_{x}^{2} e_{i}^{k+1 / 2}\right\rangle .
\end{aligned}
$$

For simplicity, the inner product in the right-hand side of 33 will be denoted by $\left\langle\xi_{1}, \xi_{2}\right\rangle$. Then $\left\langle\xi_{1}, \xi_{2}\right\rangle \leq$ $\frac{1}{2 \theta}\left\|\xi_{1}\right\|^{2}+\frac{\theta}{2}\left\|\xi_{2}\right\|^{2}$, and setting $\theta=K$, we obtain

$$
\begin{aligned}
\eta_{3} \leq & \frac{1}{2 \theta} \| \mathfrak{A}\left(c_{1}\left|\frac{3}{2} e_{i}^{k}-\frac{1}{2} e_{i}^{k-1}\right|+c_{2} \mid \frac{1}{2} e_{i}^{k+1-n}\right. \\
& \left.+\frac{1}{2} e_{i}^{k-n} \mid\right)\left\|^{2}+\frac{\theta}{2}\right\| \delta_{x}^{2} e_{i}^{k+1 / 2} \|^{2} .
\end{aligned}
$$


Recalling Lemma3, we get

$$
\begin{aligned}
\eta_{3} \leq & \frac{1}{2 \theta}\left\|c_{1}\right\| \frac{3}{2} e_{i}^{k}-\frac{1}{2} e_{i}^{k-1} \| \\
& +c_{2}\left|\frac{1}{2} e_{i}^{k+1-n}+\frac{1}{2} e_{i}^{k-n}\right|\left\|^{2}+\frac{\theta}{2}\right\| \delta_{x}^{2} e_{i}^{k+1 / 2} \|^{2} \\
\eta_{3} \leq & \frac{1}{2 \theta} h \sum_{i=1}^{M-1}\left(c_{1}\left|\frac{3}{2} e_{i}^{k}-\frac{1}{2} e_{i}^{k-1}\right|\right. \\
+ & \left.c_{2}\left|\frac{1}{2} e_{i}^{k+1-n}+\frac{1}{2} e_{i}^{k-n}\right|\right)^{2}+\frac{\theta}{2}\left\|\delta_{x}^{2} e_{i}^{k+1 / 2}\right\|^{2}, \\
\eta_{3} \leq & \frac{1}{2 \theta}\left[h c_{1}^{2} \sum_{i=1}^{M-1}\left(\frac{3}{2} e_{i}^{k}-\frac{1}{2} e_{i}^{k-1}\right)^{2}\right. \\
& \left.+c_{2}^{2} h \sum_{i=1}^{M-1}\left(\frac{1}{2} e_{i}^{k+1-n}+\frac{1}{2} e_{i}^{k-n}\right)^{2}\right] \\
& +\frac{\theta}{2}\left\|\delta_{x}^{2} e_{i}^{k+1 / 2}\right\|^{2}, \\
& +\frac{1}{2} c_{2}^{2} h \sum_{i=1}^{M-1}\left(\left(\delta_{x}^{2} e_{i}^{k+1 / 2} \|^{2},\right.\right. \\
\eta_{3} \leq & \frac{1}{2 \theta}\left[\frac{5}{2} h c_{1}^{2} \sum_{i=1}^{M-1}\left(\left(e_{i}^{k}\right)^{2}+\left(e_{i}^{k-1}\right)^{2}\right)\right. \\
& \\
& \\
&
\end{aligned}
$$

which means that

$$
\begin{aligned}
\eta_{3} \leq & \frac{1}{2 K}\left[\frac { 5 } { 2 } c _ { 1 } ^ { 2 } \left(\left(\left\|e^{k}\right\|^{2}+\left\|e^{k-1}\right\|^{2}\right)\right.\right. \\
& +\frac{1}{2} c_{2}^{2}\left(\left(\left\|e^{k+1-n}\right\|^{2}+\left\|e^{k-n}\right\|^{2}\right)\right] \\
& +\frac{K}{2}\left\|\delta_{x}^{2} e_{i}^{k+1 / 2}\right\|^{2} .
\end{aligned}
$$

Substituting by (29), (31) and (35) into 26), we get

$$
\begin{aligned}
\sum_{n=0}^{k} g_{n}^{(k+1)} & \left(\left\langle e^{n+1}, e^{n+1}\right\rangle-\left\langle e^{n}, e^{n}\right\rangle\right) \\
\leq & \frac{1}{K}\left[\frac { 5 } { 2 } c _ { 1 } ^ { 2 } \left(\left(\left\|e^{k}\right\|^{2}+\left\|e^{k-1}\right\|^{2}\right)\right.\right. \\
& \left.+\frac{1}{2} c_{2}^{2}\left(\left\|e^{k+1-n}\right\|^{2}+\left\|e^{k-n}\right\|^{2}\right)\right] \\
& +\frac{1}{K}\left\|R^{k+1 / 2}\right\|^{2}
\end{aligned}
$$

which can be written as follows:

$$
\begin{aligned}
& g_{k}^{(k+1)}\left\langle e^{k+1}, e^{k+1}\right\rangle \\
& \quad \leq \sum_{n=1}^{k}\left(g_{n}^{(k+1)}-g_{n-1}^{(k+1)}\right)\left\langle e^{n}, e^{n}\right\rangle+g_{0}^{(k+1)}\left\langle e^{0}, e^{0}\right\rangle
\end{aligned}
$$

$$
\begin{aligned}
& +\frac{1}{K}\left[\frac { 5 } { 2 } c _ { 1 } ^ { 2 } \left(\left(\left\|e^{k}\right\|^{2}+\left\|e^{k-1}\right\|^{2}\right)\right.\right. \\
& \left.+\frac{1}{2} c_{2}^{2}\left(\left\|e^{k+1-n}\right\|^{2}+\left\|e^{k-n}\right\|^{2}\right)\right] \\
& +\frac{1}{K}\left\|R^{k+1 / 2}\right\|^{2} .
\end{aligned}
$$

Since

$$
\|\nu\|^{2} \leq \frac{L^{2}}{4}\langle\nu, \nu\rangle
$$

we obtain

$$
\begin{aligned}
g_{k}^{(k+1)} & \left\langle e^{k+1}, e^{k+1}\right\rangle \\
\leq & \sum_{n=1}^{k}\left(g_{n}^{(k+1)}-g_{n-1}^{(k+1)}\right)\left\langle e^{n}, e^{n}\right\rangle \\
& +g_{0}^{(k+1)}\left\langle e^{0}, e^{0}\right\rangle+\frac{L^{2}}{4 K}\left[\frac { 5 } { 2 } c _ { 1 } ^ { 2 } \left(\left\langle e^{k}, e^{k}\right\rangle\right.\right. \\
& \left.+\left\langle e^{k-1}, e^{k-1}\right\rangle\right)+\frac{1}{2} c_{2}^{2}\left(\left\langle e^{k+1-n}, e^{k+1-n}\right\rangle\right. \\
& \left.\left.+\left\langle e^{k-n}, e^{k-n}\right\rangle\right)\right]+\frac{1}{K}\left\|R^{k+1 / 2}\right\|^{2}, \\
g_{k}^{(k+1)} & \left\langle e^{k+1}, e^{k+1}\right\rangle \\
\leq & \sum_{n=1}^{k}\left(g_{n}^{(k+1)}-g_{n-1}^{(k+1)}\right)\left\langle e^{n}, e^{n}\right\rangle \\
& +g_{0}^{(k+1)}\left\langle e^{0}, e^{0}\right\rangle+\bar{\eta}\left(\left\langle e^{k}, e^{k}\right\rangle+\left\langle e^{k-1}, e^{k-1}\right\rangle\right. \\
& \left.+\left\langle e^{k+1-n}, e^{k+1-n}\right\rangle+\left\langle e^{k-n}, e^{k-n}\right\rangle\right) \\
& +\frac{1}{K}\left\|R^{k+1 / 2}\right\|^{2}, \\
& \bar{\eta}=\frac{1}{K} \max \left\{\frac{5 c_{1} L^{2}}{8}, \frac{c_{2} L^{2}}{8}\right\} .
\end{aligned}
$$

Noting that $g_{0}^{(k+1)} \geq k_{0}>0$, and defining

$$
\begin{aligned}
E_{k}= & \max _{0 \leq l \leq k}\left\{\left\langle e^{0}, e^{0}\right\rangle\right. \\
& +\frac{\bar{\eta}}{k_{0}}\left(\left\langle e^{l}, e^{l}\right\rangle+\left\langle e^{l-1}, e^{l-1}\right\rangle\right. \\
& \left.+\left\langle e^{l+1-n}, e^{l+1-n}\right\rangle+\left\langle e^{l-n}, e^{l-n}\right\rangle\right) \\
& \left.+\frac{1}{k_{0} K}\left\|R^{l+1 / 2}\right\|^{2}\right\}
\end{aligned}
$$

we can rewrite (38) as follows:

$$
\begin{aligned}
& g_{k}^{(k+1)}\left\langle e^{k+1}, e^{k+1}\right\rangle \\
& \leq \sum_{n=1}^{k}\left(g_{n}^{(k+1)}-g_{n-1}^{(k+1)}\right)\left\langle e^{n}, e^{n}\right\rangle+g_{0}^{(k+1)} E_{k} .
\end{aligned}
$$

Using the mathematical induction, we are going to prove that

$$
\left\langle e^{k+1}, e^{k+1}\right\rangle \leq E_{k}, \quad 0 \leq k \leq \ell \leq N-1 .
$$


For $k=0$, it is easy to see that 41) can be obtained from (40). Assume that

$$
\left\langle e^{k+1}, e^{k+1}\right\rangle \leq E_{k}, \quad 0 \leq k+1 \leq r .
$$

Observing that (40), we can write

$$
\begin{aligned}
& g_{r}^{(r+1)}\left\langle e^{r+1}, e^{r+1}\right\rangle \\
& \leq \sum_{n=1}^{r}\left(g_{n}^{(r+1)}-g_{n-1}^{(r+1)}\right)\left\langle e^{n}, e^{n}\right\rangle+g_{0}^{(r+1)} E_{r} \\
& \leq \sum_{n=1}^{r}\left(g_{n}^{(r+1)}-g_{n-1}^{(r+1)}\right) E_{r}+g_{0}^{(r+1)} E_{r} \\
& =g_{r}^{(r+1)} E_{r} .
\end{aligned}
$$

Consequently, 410 is proved. Noting that $\left\langle e^{0}, e^{0}\right\rangle=$ 0 , we have

$$
\begin{aligned}
& \left\langle e^{\ell+1}, e^{\ell+1}\right\rangle \\
& \leq \frac{\bar{\eta}}{k_{0}}\left(\sum_{r=l_{0}-n}^{l_{0}-n+1}\left\langle e^{r}, e^{r}\right\rangle+\sum_{r=l_{0}-1}^{l_{0}}\left\langle e^{r}, e^{r}\right\rangle\right) \\
& \quad+\frac{1}{k_{0} K}\left\|R^{l_{0}+1 / 2}\right\|^{2},
\end{aligned}
$$

where $l_{0}$ is a number at which the maximum of $E_{\ell}$ is achieved. Since (43) fulfills all conditions of applying Lemma 9 we obtain

$$
\begin{aligned}
\left\langle e^{\ell+1}, e^{\ell+1}\right\rangle & \leq \frac{1}{k_{0} K}\left\|R^{l_{0}+1 / 2}\right\|^{2} \exp \left(\frac{4 \bar{\eta}}{k_{0}}\right) \\
& \leq C\left(\tau^{2-\alpha}+h^{4}\right)^{2}, \\
C & =\frac{L c_{3}^{2}}{k_{0} K} \exp \left(\frac{4 \bar{\eta}}{k_{0}}\right) .
\end{aligned}
$$

Recalling (44), we get

$$
\left\|e^{\ell+1}\right\|_{\infty} \leq \sqrt{\frac{3 L}{8} C\left(\tau^{2-\alpha}+h^{4}\right)^{2}} \leq \bar{C}\left(\tau^{2-\alpha}+h^{4}\right) .
$$

Thus, the inductive step for (20) is achieved and this completes the proof.

To discuss the stability of the difference scheme (11a)-11c), we also use the discrete energy method in the same way like the discussion of the convergence.

Let $\left\{\nu_{i}^{k} \mid 0 \leq i \leq M, 0 \leq k \leq N\right\}$ be the solution of

$$
\begin{aligned}
\mathfrak{A} & {\left[\omega_{1} \nu^{k}+\sum_{m=1}^{k-1}\left(\omega_{k-m+1}-\omega_{k-m}\right) \nu^{m}-\omega_{k} \nu^{0}\right.} \\
& \left.+\sigma \frac{\left(\nu_{i}^{k+1}-\nu_{i}^{k}\right)}{2^{1-\alpha}}\right] \\
= & K \delta_{x}^{2} \nu_{i}^{k+1 / 2}+\mathfrak{A} f\left(x_{i}, t_{k+1 / 2}, \frac{3}{2} \nu_{i}^{k}-\frac{1}{2} \nu_{i}^{k-1},\right. \\
& \left.\frac{1}{2} \nu_{i}^{k+1-n}+\frac{1}{2} \nu_{i}^{k-n}\right),
\end{aligned}
$$

$$
\begin{gathered}
\nu_{0}^{k}=\phi_{0}\left(t_{k}\right), \quad \nu_{M}^{k}=\phi_{L}\left(t_{k}\right), \quad 1 \leq k \leq N, \quad \\
\nu_{i}^{k}=\psi\left(x_{i}, t_{k}\right)+\rho_{i}^{k}, \quad 0 \leq i \leq M, \quad-n \leq k \leq 0
\end{gathered}
$$

where $\rho_{i}^{k}$ is the perturbation of $\psi\left(x_{i}, t_{k}\right)$.

Following the same steps as in the proof of convergence theorem, the following result is obtained.

Theorem 3. (Stability theorem) Assume that $\theta_{i}^{k}=\nu_{i}^{k}-$ $u_{i}^{k}, \quad 0 \leq i \leq M, \quad-n \leq k \leq N$. There exist constants $c_{4}, c_{5}, h_{0}, \tau_{0}$ such that

$$
\begin{gathered}
\left\|\theta^{k}\right\|_{\infty} \leq c_{4} \sqrt{\tau} \sum_{k=-n}^{0}\left\|\rho^{k}\right\|, \quad 0 \leq k \leq N, \\
\left\|\rho^{k}\right\|=\sqrt{h \sum_{i=1}^{M-1}\left(\rho_{i}^{k}\right)^{2}},
\end{gathered}
$$

provided that

$$
h \leq h_{0}, \quad \tau \leq \tau_{0}, \quad \max _{\substack{-n \leq k \leq 0 \\ 0 \leq i \leq \bar{M}}}\left|\rho_{i}^{k}\right| \leq c_{5} .
$$

\section{Numerical examples}

Let $u_{i}^{k}$ be the solution of the constructed difference scheme 111a-111c with the step sizes $\tau$ and $h$. Define the maximum norm error by

$$
E(\tau, h)=\max _{\substack{0 \leq i \leq M \\ 0 \leq k \leq N}}\left\|U_{i}^{k}-u_{i}^{k}\right\|_{\infty}
$$

Define the following error rates:

$$
\begin{aligned}
& \text { rate }_{1}=\log _{2}\left(\frac{E(2 \tau, h)}{E(\tau, h)}\right), \\
& \text { rate }_{2}=\log _{2}\left(\frac{E(\tau, 2 h)}{E(\tau, h)}\right) .
\end{aligned}
$$

Example 1. Consider the following test example:

$$
\begin{gathered}
\frac{\partial^{\alpha} u(x, t)}{\partial t^{\alpha}}=\frac{\partial^{2} u(x, t)}{\partial x^{2}}+f(x, t, u(x, t), u(x, t-s)) \\
\quad t \in(0,1), \quad 0<x<2, \quad \text { (48) } \\
f(x, t, u(x, t), u(x, t-s) \\
=\frac{\Gamma(3)}{\Gamma(3-\alpha)}\left(2 x-x^{2}\right) t^{2-\alpha} \\
+2 t^{2}-u(x, t-s)+x(2-x)(t-s)^{2},
\end{gathered}
$$

with the initial and boundary conditions

$$
\begin{gathered}
u(x, t)=t^{2}\left(2 x-x^{2}\right), \quad 0 \leq x \leq 2, \quad t \in[-s, 0), \\
u(0, t)=u(2, t)=0, \quad t \in[0,1] .
\end{gathered}
$$

The exact solution to this problem is

$$
u(x, t)=t^{2}\left(2 x-x^{2}\right) .
$$


Example 2. Consider the following test example:

$$
\begin{gathered}
\frac{\partial^{\alpha} u(x, t)}{\partial t^{\alpha}}=\frac{\partial^{2} u(x, t)}{\partial x^{2}}+f(x, t, u(x, t), u(x, t-s)), \\
\quad t \in(0,1), \quad 0<x<1, \quad(52) \\
f(x, t, u(x, t), u(x, t-s) \\
=\frac{\Gamma(7 / 2)}{\Gamma(7 / 2-\alpha)}\left(x^{2}-x\right) t^{5 / 2-\alpha}-2 t^{5 / 2} \\
+u^{2}(x, t-s)-\left(x^{2}-x\right)^{2}(t-s)^{5}
\end{gathered}
$$

with the initial and boundary conditions

$$
\begin{gathered}
u(x, t)=t^{\frac{5}{2}}\left(x-x^{2}\right), \quad 0 \leq x \leq 1, \quad t \in[-s, 0) \\
u(0, t)=u(1, t)=0, \quad t \in[0,1]
\end{gathered}
$$

The exact solution to this problem is

$$
u(x, t)=t^{\frac{5}{2}}\left(x^{2}-x\right) .
$$

Tables 1, 2 and 3, 4 show the errors in maximum norm and their convergence rates for time fractional models 48 51 and 52 55 respectively. From these tables, it can be seen that the orders of convergence of the proposed numerical method are in good agreement with the theoretical results in the theorem.

Table 1. Errors and convergence orders of the difference scheme 11a)-11c in the time variable with $h=1 / 300$ and $\alpha=0.25$ with time delay $s=1$.

\begin{tabular}{|c|c|c|}
\hline$\tau$ & $E(\tau, h)$ & rate $_{1}$ \\
\hline \hline$\frac{1}{10}$ & 0.00113 & \\
$\frac{1}{20}$ & 0.00034 & 1.735 \\
$\frac{1}{40}$ & 0.0001 & 1.742 \\
$\frac{1}{80}$ & 0.00003 & 1.748 \\
$\frac{1}{160}$ & 0.000009 & 1.752 \\
\hline
\end{tabular}

Table 2. Errors and convergence orders of the difference scheme (11a)-11c) in the space variable with $\tau=1 / 1000$ and $\alpha=0.25$ with time delay $s=1$.

\begin{tabular}{|c|c|c|}
\hline$h$ & $E(\tau, h)$ & rate $_{2}$ \\
\hline \hline$\frac{1}{4}$ & 0.0025 & \\
$\frac{1}{8}$ & 0.00017 & 3.863 \\
$\frac{1}{16}$ & 0.00001 & 3.895 \\
$\frac{1}{32}$ & 0.0000007 & 3.942 \\
$\frac{1}{64}$ & 0.0000004 & 3.975 \\
\hline
\end{tabular}

Table 3. Errors and convergence orders of the difference scheme 11a)-11c in the time variable with $h=1 / 500$ and $\alpha=0.75$ with time delay $s=0.5$.

\begin{tabular}{|c|c|c|}
\hline$\tau$ & $E(\tau, h)$ & rate $_{1}$ \\
\hline \hline$\frac{1}{10}$ & 0.00112 & \\
$\frac{1}{20}$ & 0.00047 & 1.245 \\
$\frac{1}{40}$ & 0.00019 & 1.248 \\
$\frac{1}{80}$ & 0.00008 & 1.249 \\
$\frac{1}{160}$ & 0.00003 & 1.252 \\
\hline
\end{tabular}

Table 4. Errors and convergence orders of the difference scheme 11a- 11c in the space variable with $\tau=1 / 2000$ and $\alpha=0.75$ with time delay $s=0.5$.

\begin{tabular}{|c|c|c|}
\hline$h$ & $E(\tau, h)$ & rate $_{2}$ \\
\hline \hline$\frac{1}{4}$ & 0.0121 & \\
$\frac{1}{8}$ & 0.00076 & 3.980 \\
$\frac{1}{16}$ & 0.00005 & 3.995 \\
$\frac{1}{32}$ & 0.000003 & 3.998 \\
$\frac{1}{64}$ & 0.0000001 & 4.010 \\
\hline
\end{tabular}

\section{Conclusion}

The main contribution of this work lies in building a linearized difference scheme to solve a class of time fractional diffusion equations with non-linear delay. We proved that our scheme is unconditionally convergent and stable in the sense of the maximum norm. In our future work, we plan to increase the time convergence order to two instead of $2-\alpha, 0<\alpha \leq 1$, by using a suitable approximation for the time Caputo fractional derivative in the problem under consideration. The proposed numerical test examples supported our theoretical results.

\section{Acknowledgment}

This work was supported by the Government of Russian Federation under the grant no. 02.A03.21.0006.

\section{References}

Alikhanov, A.A. (2015). A new difference scheme for the time fractional diffusion equation, Journal of Computational Physics 280: 424-438.

Bagley, R.L. and Torvik, P.J. (1983). A theoretical basis for the application of fractional calculus to viscoelasticity, Journal of Rheology 27(201): 201-210.

Balachandran, K. and Kokila, J. (2012). On the controllability of fractional dynamical systems, International Journal of Applied Mathematics and Computer Science 22(3): 523-531, DOI: 10.2478/v10006-012-0039-0.

Batzel, J.J. and Kappel, F. (2011). Time delay in physiological systems: Analyzing and modeling its impact, Mathematical Biosciences 234(2): 61-74. 
Bellen, A. and Zennaro, M. (2003). Numerical Methods for Delay Differential Equations, Oxford University Press, Oxford.

Benson, D., Schumer, R., Meerschaert, M.M. and Wheatcraft, S.W. (2001). Fractional dispersion, Levy motion, and the made tracer tests, Transport in Porous Media 42(1-2): 211-240.

Chen, F. and Zhou, Y. (2011). Attractivity of fractional functional differential equations, Computers and Mathematics with Applications 62(3): 1359-1369.

Culshaw, R. V., Ruan, S. and Webb, G. (2003). A mathematical model of cell-to-cell spread of HIV-1 that includes a time delay, Mathematical Biology 46: 425-444.

Ferreira, J.A. (2008). Energy estimates for delay diffusion-reaction equations, Computational and Applied Mathematics 26(4): 536-553.

Hao, Z., Fan, K., Cao, W. and Sun, Z. (2016). A finite difference scheme for semilinear space-fractional diffusion equations with time delay, Applied Mathematics and Computation 275: $238-254$.

Hatano, Y. and Hatano, N. (1998). Dispersive transport of ions in column experiments: An explanation of long-tailed profiles, Water Resources Research 34(5): 1027-1033.

Höfling, F. and Franosch, T. (2013). Anomalous transport in the crowded world of biological cells, Reports on Progress in Physics 76(4): 46602.

Holte, J.M. (2009). Discrete Gronwall lemma and applications, MAA North Central Section Meeting at UND, Grand Forks, $N D, U S A$, p. 8, http://homepages.gac.edu/ nolte/publications/GronwallLemma.pdf

Jackiewicz, Z., Liu, H., Li, B. and Kuang, Y. (2014). Numerical simulations of traveling wave solutions in a drift paradox inspired diffusive delay population model, Mathematics and Computers in Simulation 96: 95-103.

Karatay, I., Kale, N. and Bayramoglu, S.R. (2013). A new difference scheme for time fractional heat equations based on Crank-Nicholson method, Fractional Calculus and Applied Analysis 16(4): 893-910.

Kruse, R. and Scheutzow, M. (2016). A discrete stochastic Gronwall lemma, Mathematics and Computers in Simulation, DOI: 10.1016/j.matcom.2016.07.002.

Lakshmikantham, V. (2008). Theory of fractional functional differential equations, Nonlinear Analysis: Theory, Methods and Applications 69(10): 3337-3343.

Lekomtsev, A. and Pimenov, V. (2015). Convergence of the scheme with weights for the numerical solution of a heat conduction equation with delay for the case of variable coefficient of heat conductivity, Applied Mathematics and Computation 256: 83-93.

Liu, P.-P. (2015). Periodic solutions in an epidemic model with diffusion and delay, Applied Mathematics and Computation 265: 275-291.

Meerschaert, M.M. and Tadjeran, C. (2004). Finite difference approximations for fractional advection-dispersion flow equations, Computational and Applied Mathematics 172(1): 65-77.
Miller, K.S. and Ross, B. (1993). An Introduction to the Fractional Calculus and Fractional Differential Equations, Miller, New York, NY.

Pimenov, V.G. and Hendy, A.S. (2015). Numerical studies for fractional functional differential equations with delay based on BDF-type shifted Chebyshev polynomials, $A b$ stract and Applied Analysis, 2015(2015), Article ID 510875, DOI: $10.1155 / 2015 / 510875$.

Pimenov, V.G., Hendy, A.S. and De Staelen, R.H. (2017). On a class of non-linear delay distributed order fractional diffusion equations, Journal of Computational and Applied Mathematics 318: 433-443.

Raberto, M., Scalas, E. and Mainardi, F. (2002). Waiting-times returns in high frequency financial data: An empirical study, Physica A 314(1-4): 749-755.

Ren, J. and Sun, Z.Z. (2015). Maximum norm error analysis of difference schemes for fractional diffusion equations, $A p$ plied Mathematics and Computation 256: 299-314.

Rihan, F.A. (2009). Computational methods for delay parabolic and time-fractional partial differential equations, Numerical Methods for Partial Differential Equations 26(6): 1557-1571.

Samarskii, A.A. and Andreev, V.B. (1976). Finite Difference Methods for Elliptic Equations, Nauka, Moscow, (in Russian).

Scalas, E., Gorenflo, R. and Mainardi, F. (2000). Fractional calculus and continuous-time finance, Physica A 284(1-4): 376-384.

Schneider, W. and Wyss, W. (1989). Fractional diffusion and wave equations, Journal of Mathematical Physics 30(134): 134-144.

Sikora, B. (2016). Controllability criteria for time-delay fractional systems with a retarded state, International Journal of Applied Mathematics and Computer Science 26(3): 521-531, DOI: 10.1515/amcs-2016-0036.

Solodushkin, S.I., Yumanova, I.F. and De Staelen, R.H. (2017). A difference scheme for multidimensional transfer equations with time delay, Journal of Computational and Applied Mathematics 318: 580-590.

Tumwiine, J., Luckhaus, S., Mugisha, J.Y.T. and Luboobi, L.S. (2008). An age-structured mathematical model for the within host dynamics of malaria and the immune system, Journal of Mathematical Modelling and Algorithms 7: 79-97.

Wyss, W. (1986). The fractional diffusion equation, Journal of Mathematical Physics 27: 2782-2785.

Yan, Y. and Kou, C. (2012). Stability analysis of a fractional differential model of HIV infection of CD4+ T-cells with time delay, Mathematics and Computers in Simulation 82(9): 1572-1585.

Zhang, Z.B. and Sun, Z.Z. (2013). A linearized compact difference scheme for a class of nonlinear delay partial differential equations, Applied Mathematical Modelling 37(3): 742-752. 


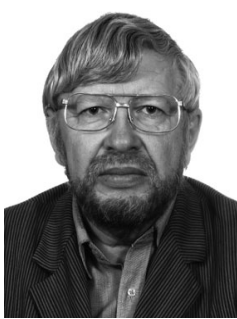

Vladimir Pimenov holds a DSc degree in physics and mathematics and is a professor and the head of the Department of Computational Mathematics, Ural Federal University, Ekaterinburg, Russia. His current research activities focus on numerical methods for the solution of functional differential equations, partial differential equations with delay, fractional functional differential equations and the theory of the positional control of systems with delay.

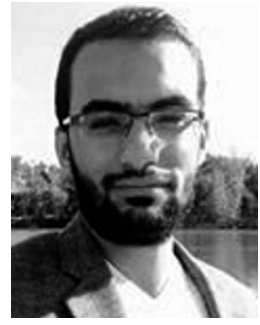

Ahmed Hendy is a junior researcher and a $\mathrm{PhD}$ student in the Department of Computational Mathematics, Ural Federal University, Ekaterinburg, Russia. He is also a teaching assistant in the Department of Mathematics, Faculty of Science, Benha University, Egypt. His current research activities focus on numerical solution of fractional partial differential equations with delay based on difference methods and their theoretical analysis.

Received: 29 November 2016

Revised: 21 April 2017

Re-revised: 3 May 2017

Accepted: 4 May 2017 